\title{
Biomimetic Electro-oxidation of Alkyl Sulfides from Exfoliated Molybdenum Disulfide Nanosheets
}

Received 00th January 20xx, Accepted 00th January 20xx DOI: $10.1039 / x 0 x \times 00000 x$

\author{
Lahcene Maachou, ${ }^{\mathrm{a}+}$ Kun Qi, ${ }^{a+}$ Eddy Petit, ${ }^{\mathrm{a}}$ Zhaodan Qin, ${ }^{\mathrm{a}}$ Yang Zhang, ${ }^{\mathrm{a}}$ Didier Cot, ${ }^{\mathrm{a}}$ Valérie Flaud, ${ }^{\mathrm{b}}$ \\ Corine Reibel, ${ }^{\mathrm{b}}$ Heba El-Maghrabi, ${ }^{a}$ Lei Li, ${ }^{c}$ Philippe Miele, ${ }^{a}$ Daniel Kaplan, ${ }^{d}$ Manish Chhowalla, ${ }^{e}$ \\ Nicolas Onofrio, ${ }^{f}$ Damien Voiry*a
}

\begin{abstract}
Enzymes are biological catalysts that are interesting for key reactions such as hydrogen evolution, $\mathrm{CO}_{2}$ conversion into hydrocarbons and the fixation of nitrogen. Enzymes are particularly good catalysts for organic reactions because of their high selectivity. However, they exhibit modest stability and require extensive purification, which makes them costly. Here, we report a biomimetic electroactive two-dimensional (2D) catalyst based on single-layer metallic MoS 2 nanosheets for the oxidation of alkyl and aryl sulfides. The structure of the $\mathrm{MoS}_{2}$ nanosheets mimics the active site of natural dimethyl sulfoxide (DMSO) reductase found in anaerobic bacteria. We demonstrate that 2D $\mathrm{MoS}_{2}$ nanosheets efficiently oxidize organic sulfides. Notably, we show that dimethyl sulfide can be electro-oxidized to DMSO with activity surpassing that of noble metal catalysts. The production of DMSO using metallic $1 T^{\prime}$ phase $\mathrm{MoS}_{2}$ reaches $680 \mathrm{~L} \mathrm{~h}^{-1}$ per gram at $1500 \mathrm{mV}$ vs. NHE - 4 times higher than platinum nanoparticles - and is stable for $>24$ hours. Our findings provide new directions for electrosynthesis from metallic 2D materials.
\end{abstract}

\section{Introduction}

Enzymes are proteins that catalyze key reactions in living organisms. ${ }^{1}$ They possess high selectivity, which makes them attractive for enantioselective synthesis of drug molecules in the pharmaceutical industry. ${ }^{2,3}$ The catalytically active sites on enzymes typically consist in coordinated transition metals and recent insights into how they catalyze reactions has enabled progress in the design of novel bio-inspired electrocatalysts. ${ }^{4}$ For example, understanding of mechanisms that are responsible for hydrogenase activity has allowed the development of electro-active molecular catalysts with structures that reproduce active sites of the natural enzyme. ${ }^{5}$ Nanozymes are nanomaterials with enzyme-like characteristics with superior stability compared to natural proteins - making them attractive as electro-catalysts with tunable activity. ${ }^{6,7}$ Since the discovery of unexpected peroxidase-like activity of iron oxide nanoparticles, ${ }^{8}$ various nanozymes have been

Institut Européen des Membranes, IEM, UMR 5635, University of Montpellier, ENSCM, CNRS, 34095 Montpellier Cedex5, France Institut Charles Gerhardt, ICGM, UMR 5253, University of Montpellier, ENSCM, CNRS, 34095 Montpellier Cedex5, France

College of Biological, Chemical Sciences and Engineering, Jiaxing University, Jiaxing, Zhejiang 314001, China

US Army CCDC-AC RDECOM-ARDEC, Advanced Materials Technology Branch, Picatinny Arsenal, New Jersey 07806, USA

Department of Materials Science \& Metallurgy, University of

Cambridge, Cambridge, UK

Department of Applied Physics, The Hong Kong Polytechnic University, Hong Kong, China

† These authors contributed equally.

Electronic Supplementary Information (ESI) available: [details of any supplementary information available should be included here]. See DOI: 10.1039/x0xx00000x realized using metal oxides, noble metals, graphene and derivatives, and metal-organic frameworks (MOFs). 9-11 However, two outstanding challenges remain: first, nanozymes show much lower catalytic activity because of the low densities of active sites; ${ }^{12}$ and second, the inhomogeneous composition and crystal facet structure ${ }^{13}$ make it difficult to identify the active sites. These bottlenecks are significant hurdles for implementation of nanozymes in applications.

Exfoliated two-dimensional (2D) materials are known to be good electrocatalysts. ${ }^{14}$ In particular, transition metal dichalcogenides (TMDs) have been widely studied for their catalytic properties in reactions such as the hydrogen evolution reaction, as well as oxygen and $\mathrm{CO}_{2}$ reduction reactions. ${ }^{15-17}$ Despite the large body of work on catalysis with 2D materials, their application in electrosynthesis of organic molecules has yet to be explored. Oxidation of organic sulfide compounds into sulfoxides for production of drugs such as esomeprazole and armodafinil are important reactions in the pharmaceutical industry. ${ }^{18,19}$ With an annual production of approaching 100,000 metric tons, dimethyl sulfoxide (DMSO) is widely used as polar and aprotic organic solvent in pharmaceutical and microelectronic industry as well as an intermediate for the synthesis of sulfur-based derivative molecules. Contrary to thiols, organic sulfide are stable in air and the industrial process for the synthesis of sulfoxide relies on the use of undesirable strong oxidizing agents including $\mathrm{NO}_{x}$ or peroxy acids. ${ }^{20,21}$ Redox processes can be used to oxidize organic sulfides at room temperature and without the use of harsh chemicals - greatly limiting the safety concerns. However, only handful contributions have been reported on the electro-oxidation of DMS in dimethylsulfoxide and dimethylsulfone using platinum 
as anode. ${ }^{22,23}$ The use of expensive and scarce noble metals therefore limits practical applications.

In this work, we report metallic two-dimensional $\mathrm{MoS}_{2}$ as biomimietic catalyst with intrinsic enzyme-like active sites for the electro-oxidation of organic sulfides. The atomic structure of $\mathrm{MoS}_{2}$ resembles that of the DMSO reductase active site - an enzyme responsible for reversible reduction of DMSO in DMS. When tested in oxygen-saturated, non-aqueous electrolyte solution, DMS is electrochemically converted to DMSO on the $\mathrm{MoS}_{2}$ nanosheets with activity and selectivity superior to those of noble metals. Our results show that the production of DMSO from the electrochemical oxidation of DMS using $1 \mathrm{~T}^{\prime} \mathrm{MoS}_{2}$ nanosheet catalysts is significantly higher ( $680 \mathrm{~L} \mathrm{h-1}$ per gram) than for Pt nanoparticle catalysts $\left(210 \mathrm{~L} \mathrm{~h}^{-1} \mathrm{~g}^{-1}\right)$. Our numerical predictions suggest that the superior activity is ascribed to the lower binding energy of DMS on the basal plane and the edge sites of the $1 T^{\prime}$ phase.

\section{Results and discussion}

\section{Chemically exfoliated $\mathrm{MoS}_{2}$ nanosheets}

$\mathrm{MoS}_{2}$ nanocrystals are known catalysts for the oxidation of sulfur-based molecules. For example, Lauristen et al. have observed thiophene adsorption at the brim sites on sulfide $\mathrm{MoS}_{2}$ crystals, suggesting that such sites can play an active role in catalysis. ${ }^{24}$ Catalytic oxidation of thiols into disulfides has also been achieved - hinting at nanozyme-like activity of $\mathrm{MoS}_{2}$ for oxidation of sulfur-based molecules. ${ }^{25}$ Recently $\mathrm{MoS}_{2}$ with inclusion of cobalt single-atom have been proposed for the heterogeneous oxidation of organic sulfide using $\mathrm{H}_{2} \mathrm{O}_{2}$ as oxidizing agent. ${ }^{26}$ Alternatively, DMSO can be prepared from the oxidation of DMS with a standard potential of $574 \mathrm{mV}$ vs. NHE in water but the reaction suffers from large overpotential and possible degradation of the catalyst materials (Figure S1). ${ }^{27}$ The similarity between the active sites of hydrogenase and the structure of $\mathrm{MoS}_{2}$ edges led to its use as an HER catalyst. ${ }^{5}$ DMSO reductase (DMSOR) - an enzyme produced under anaerobic conditions in some bacteria - is known to reversibly reduce DMSO into DMS. In contrast to hydrogenase that contains Fe and Mo metals, DMSOR active site consists only of molybdenum $\mathrm{Mo}(\mathrm{VI})$ coordinated with 4 sulfur atoms from two pyranopterin dithiolene ligands (Figures 1a,b). We synthesized the $1 \mathrm{~T}^{\prime}$ and $2 \mathrm{H}$ $\mathrm{MoS}_{2}$ nanosheets using our previously reported intercalationassisted exfoliation method (Figure 1c,d) (See Experimental Section). ${ }^{28,29}$ The nanosheets were exfoliated in water and first characterized by atomic force microscopy (Figure 1e). The size distributions extracted from the AFM measurements reveal that the nanosheets are $\approx 200 \mathrm{~nm}$ in length while the average thickness is $\leq 1 \mathrm{~nm}$, consistent with single layer nanosheets (Figure 1f). It is well known that as-synthesized nanosheets by chemical exfoliation possess the $1 \mathrm{~T}^{\prime}$ phase structure. ${ }^{30}$ The $2 \mathrm{H}$ phase is obtained by mild annealing (at $300^{\circ} \mathrm{C}$ under $5 \% \mathrm{H}_{2}$ in argon, See Experimental Section) of the $1 \mathrm{~T}^{\prime}$ phase $\mathrm{MoS}_{2}$ nanosheets) of the as-synthesized nanosheets. ${ }^{29,30}$ From the deconvolution of the XPS spectra
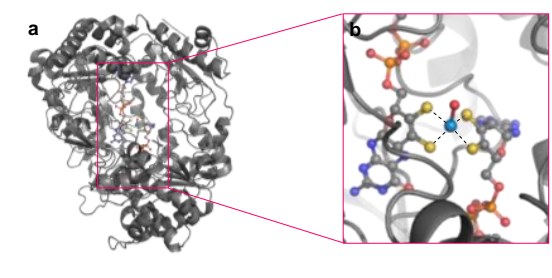

c $2 \mathrm{HMOS}_{2}$

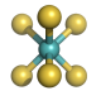

d
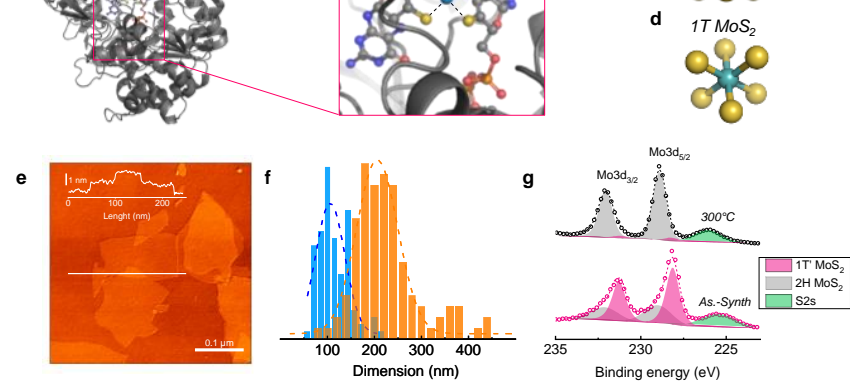

Figure 1. Characterization of chemically exfoliated $\mathrm{MoS}_{2}$ nanosheets. (a) Structural model of DMSO reductase enzyme. (b) Structure of the active sites of the DMSO reductase showing the molybdenum atom coordinated with two pyranopterin dithiolene ligands. Color code for the atoms: grey: $C$, green: Mo, yellow: $S$, orange: $P$, red: $O$, blue: $N$. (c,d) $2 \mathrm{H}$ and $1 T^{\prime}$ phase of $\mathrm{MoS}_{2}$ with Mo atoms in a trigonal prismatic and octahedral coordination with sulfur atoms. (e) Mixed topographic and phase image obtained by using atomic force microscopy (AFM). Inset: height profile of an individual exfoliated $\mathrm{MoS}_{2}$ nanosheet deposited on $300 \mathrm{~nm} \mathrm{SiO} / \mathrm{Si}$ wafer. (f) Length (orange) and width (blue) distributions of the exfoliated $\mathrm{MoS}_{2}$ nanosheets measured by AFM. The Gaussian fits of the length and width distribution are shown as dashed line. $(g) X$-ray photoelectron spectroscopy of the Mo $3 d$ regions of as-exfoliated and $300^{\circ} \mathrm{C}$-annealed $\mathrm{MoS}_{2}$ nanosheets. The raw and the fitted data are shown with open symbols and dashed lines respectively. From the deconvolution of the raw XPS signals, the metallic $1 T^{\prime}$ polymorph composes $70 \%$ of as-exfoliated nanosheets. At the opposite, the annealed nanosheets consist in $95 \%$ of the semiconducting $2 \mathrm{H}$ polymorph.

from the Mo3d and S2p regions, we have estimated the $1 T^{\prime}$ phase concentration in the as-exfoliated nanosheets to be $\approx$ $75 \%$, while the $2 \mathrm{H}$ phase is almost entirely restored after annealing. ${ }^{29}$ The presence of the $1 \mathrm{~T}^{\prime}$ and $2 \mathrm{H}$ phases is also detected in Raman of as-exfoliated and annealed $\mathrm{MoS}_{2}$ nanosheets (Figure S2).

The catalysts were prepared by loading a controlled amount of $1 \mathrm{~T}^{\prime}$ phase $\mathrm{MoS}_{2}$ nanosheets on the electrode via drop casting and controlled evaporation of the solvent. ${ }^{[29]}$ Scanning electron microscopy (SEM) on the electrode reveals that the nanosheets are densely packed on the surface without significant porosity (Figure 2a). The $2 \mathrm{H} \mathrm{MoS}_{2}$ electrode is virtually identical (Inset Figure 2a) - meaning that the phase transition during mild annealing does not affect the orientation or morphology of thenanosheets so that only the phase of $\mathrm{MoS}_{2}$ is modified. Next, we determined the electrochemically active surface area (ECSA) by estimating the double layer capacitance $\left(C_{d l}\right)$ of $1 T^{\prime}$ - and $2 \mathrm{H}$ $\mathrm{MoS}_{2}$ electrodes (See "Estimation of the double layer capacitance for $\mathrm{MoS}_{2}$ nanosheets and Pt nanoparticles" in the Experimental Section). To do this, different electrodes were cycled in 0.1M tetraethylammonium tetrafluoroborate $\left(\mathrm{NEt}_{4} \mathrm{BF}_{4}\right)$ in acetonitrile at increasing scan rates from 5 to 200 $\mathrm{mV} \mathrm{s}^{-1}$ (Figure $\mathbf{2 b}, \mathbf{c}$ ). The double layer capacitances from the $1 \mathrm{~T}^{\prime}$ and $2 \mathrm{H}$ electrodes were found to be 284 and $55 \mu \mathrm{F} \mathrm{cm}{ }^{-2}$, respectively (Figure $2 \mathrm{~d}$ ). Assuming a $\mathrm{C}_{\mathrm{dl}}$ of $\approx 10 \mu \mathrm{F} \mathrm{cm}^{-2}$ for ideally flat $\mathrm{MoS}_{2}$ in organic electrolytes, ${ }^{32}$ the ECSA is estimated to $28.4 \mathrm{~cm}_{\mathrm{ECSA}}^{-2}$ and $5.5 \mathrm{~cm}_{\mathrm{ECSA}}^{-2}$ for electrodes with $1 \mathrm{~T}^{\prime}$ and $2 \mathrm{H}$ phases, respectively. The reduction of the ECSA values between $1 \mathrm{~T}^{\prime}$ and $2 \mathrm{H}$ phases $\mathrm{MoS}_{2}$ is likely due to the decrease in the interlayer spacing of the nanosheets after annealing and therefore limited intercalation of ions in the $2 \mathrm{H} \mathrm{MoS}_{2}$ electrodes as reported in 

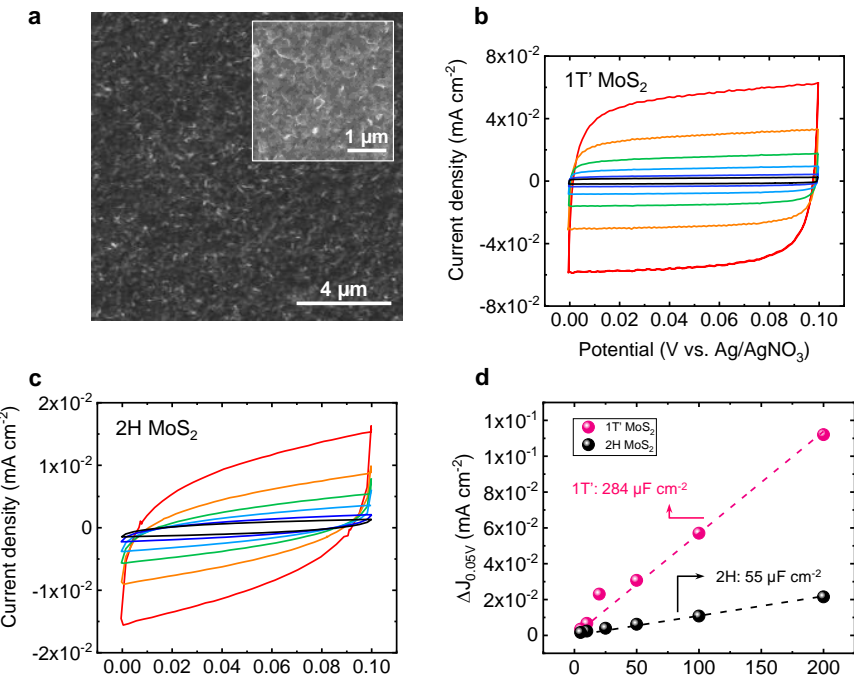

Potential ( $\mathrm{V}$ vs. $\mathrm{Ag} / \mathrm{AgNO}_{3}$ )

d

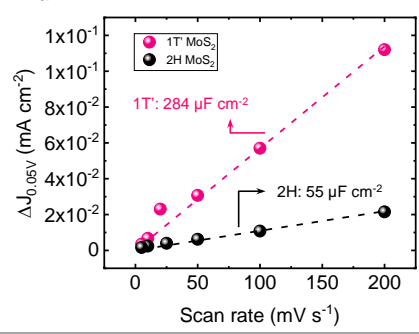

Figure 2. Characterization of the MoS electrodes. (a) Top view of the MoS electrodes observed under scanning electron microscope (SEM). (b,c) Cyclic voltammetry curves of the $1 T^{\prime}$ phase (b) and the $2 \mathrm{H}$ phase (c) of $\mathrm{MOS}_{2}$ nanosheets deposited on glassy carbon electrodes measured at increasing scan rates from 5 to $200 \mathrm{mV} \mathrm{s}^{-1}$. (d) Corresponding evolution of $\Delta J_{0.05 \mathrm{~V}}$ with the scan rates for the $1 T^{\prime}$ and $2 \mathrm{H}$ phase $\mathrm{MoS}_{2}$. The slope gives access to the double layer capacitance $\left(C_{d l}\right)$ of the $\mathrm{MoS}_{2}$ electrodes.

Ref . ${ }^{33}$ The $C_{d l}$ is also significantly lower in organic electrolyte than in aqueous electrolytes (Figure S3) due to nature of electrochemically active sites being different for different types of electrolyte solutions.

\section{Electrochemical activity of $\mathrm{MoS}_{2}$ nanosheets towards the oxidation of DMS}

The electrochemical responses from $1 \mathrm{~T}^{\prime}$ and $2 \mathrm{H} \mathrm{MoS}_{2}$ nanozymes were measured in $0.1 \mathrm{M} \mathrm{NEt}_{4} \mathrm{BF}_{4}$ in acetonitrile and the applied potentials corrected versus Normal Hydrogen Electrode (NHE) after calibrating the reference electrode using ferrocene: $\mathrm{Fc}_{\mathrm{Fc}}{ }^{+}$(Figure S5). The electrolyte solution was saturated with $\mathrm{O}_{2}$ used as a source of oxygen for the reaction. When sweeping the potential of the working electrodes, the anodic current density rapidly and continuously increased for both phases of $\mathrm{MoS}_{2}$ nanosheets (Figure 3a). The onset potential (the potential at which the Faradaic current begins to a

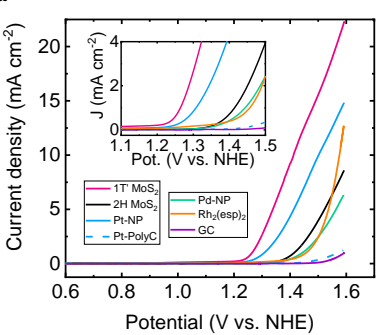

b

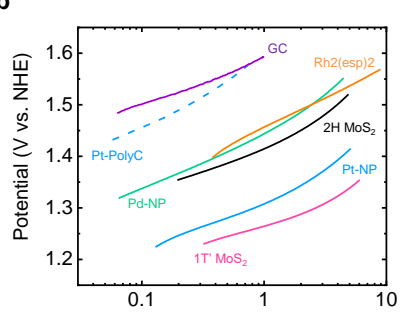

Current density $\left(\mathrm{mA} \mathrm{cm}^{-2}\right)$
Figure 3. Electrocatalytic behavior of $\mathrm{MoS}_{2}$ electrodes towards the oxidation of DMS compared with reference catalysts. (a) Polarization curves of $1 T^{\prime}$ and $2 \mathrm{H} \mathrm{MoS}_{2}$ nanosheets in presence DMS compared to platinum nanoparticles, polycrystalline platinum, palladium nanoparticles, $\mathrm{Rh}_{2}(\mathrm{esp})_{2}$ and glassy carbon. Inset: Magnification of the low potential region. Scan rate: $20 \mathrm{mV} \mathrm{s}^{-1}$. (b) Corresponding Tafel plots from the different $\mathrm{MoS}_{2}$ electrodes compared to platinum. The smallest values of Tafel slopes are obtained for $1 \mathrm{~T}^{\prime} \mathrm{MoS}_{2}$ nanosheets with $75 \mathrm{mV} \mathrm{dec}^{-1}$. increase) from the $1 \mathrm{~T}^{\prime}$ phase was found to be $1190 \mathrm{mV}$ vs. NHE - clearly lower than the onset potential of $2 \mathrm{H}$ phase of $\mathrm{MoS}_{2}$ (1280 mV vs. NHE). To confirm that the anodic current is solely due to the oxidation of DMS, we performed the same experiment but in the absence of DMS. Virtually no current wasdetected without - confirming that the anodic current is due to the reaction of DMS and not the oxidation of the catalyst itself (Figure S6). To further quantify the catalytic performance of the $\mathrm{MoS}_{2}$ nanosheets with respect to state-of-the art catalysts, we compare the results with 60 mesh palladium: PdNP, Polycrystalline platinum: Pt-PolyC, commercial platinum nanoparticles supported on porous carbon ( $20 \%$ in mass): $P d-$ $\mathrm{NP}$ and a dirhodium(II) carboxylate complex: $\mathrm{Rh}_{2}$ (esp) ${ }_{2}(\mathrm{esp}=\alpha$, $\alpha^{\prime}, \alpha^{\prime}, \alpha^{\prime}$-tetramethyl-1,3-benzenedipropionic acid) that is a known catalysts for the oxidation of organic sulfides. ${ }^{34}$ The results reveal that the $1 T^{\prime}$ phase of $\mathrm{MoS}_{2}$ catalysts exhibit geometrical current density of $9.2 \mathrm{~mA} \mathrm{~cm}_{\text {geom }}^{-2}$ at $1400 \mathrm{mV}$ vs. NHE compared to $4.3 \mathrm{~mA} \mathrm{~cm}$ geom for Pt nanoparticle electrodes (Figure 3a). 1T' $\mathrm{MoS}_{2}$ catalysts also outperforms $\mathrm{Pt}$ nanoparticles, palladium and dirhodium complex with a lower onset potential of $\approx 45 \mathrm{mV}, 150 \mathrm{mV}$ and $160 \mathrm{mV}$ respectively (Inset Figure 3a). The substantially better electrocatalytic activity of $1 \mathrm{~T}^{\prime}$ phase $\mathrm{MoS}_{2}$ is also supported by Tafel plots shown in Figure 3b. The Tafel slope of $1 \mathrm{~T}^{\prime} \mathrm{MoS}_{2}$ reaches $75 \mathrm{mV} \mathrm{dec}^{-1}$. For comparison Tafel slopes of $99 \mathrm{mV} \mathrm{dec}^{-1}, 110 \mathrm{mV} \mathrm{dec}^{-1}, 125$ and $115 \mathrm{mV} \mathrm{dec}^{-1}$ are obtained for $2 \mathrm{H} \mathrm{MoS}_{2}$, Pt nanoparticles, $\mathrm{Pd}$ and dirhodium respectively. The reduced Tafel slope is attributed to the improved electrocatalytic reaction kinetics due to higher electrical conductivity of metallic $1 T^{\prime}$ phase of $\mathrm{MoS}_{2}{ }^{31,35}$

To assess the true electrocatalytic activity of $\mathrm{MoS}_{2}$ nanosheets for the reduction of DMS, we normalized the geometrical current to the ECSA values obtained from the $C_{d l}$ measurements. We determined the surface of platinum exposed to the electrolyte for both polycrystalline $\mathrm{Pt}$ and $\mathrm{Pt}$ nanoparticles using the copper underpotential deposition ( $\mathrm{Cu}$ UPD) method developed by Green and Kucernak. ${ }^{36}$ Under UPD conditions at $+0.3 \mathrm{~V}$ vs. $\mathrm{RHE}, \mathrm{Cu}$ is solely deposited on the active Pt while no copper is deposited on the carbon support. The density of active sites on Pt was determined from the exchanged charges when stripping $\mathrm{Cu}$ monolayers deposited on Pt nanoparticles: Qstrip. Assuming $420 \mu \mathrm{C} \mathrm{cm}^{-2}$ of charges exchanged for the deposition of a monolayer of $\mathrm{Cu}$ on $\mathrm{Pt},{ }^{36}$ the active surfaces for polycrystalline $\mathrm{Pt}$ and $\mathrm{Pt}$ nanoparticles were estimated to 4.14 and $14.8 \mathrm{~cm}^{2} / \mathrm{cm}_{\text {Geom }}^{2}$ (" $\mathrm{Cu}$ Underpotential Deposition" section in the Supporting Information and Figure S7). We note that while the Cu UPD method has been developed for unsupported platinum, we found that the surface area of the Pt nanoparticles reaches $10 \mathrm{~m}^{2} \mathrm{~g}^{-1}$ of catalyst, which is close to the values for unsupported Pt nanoparticles. ${ }^{36}$ Figure 4a shows the ECSA-normalized current density $\left(J_{E C S A}=\frac{J_{\text {Geom }}}{E C S A}\right)$ of the different electrodes. The normalized current density for $1 \mathrm{~T}^{\prime}$ and $2 \mathrm{H} \mathrm{MoS}_{2}$ reach 0.32 and $0.13 \mathrm{~mA} \mathrm{~cm}_{E C S A}^{-2}$ at $1400 \mathrm{mV}$ vs. NHE respectively. A lower onsetpotential is observed from the $1 \mathrm{~T}^{\prime}$ $\mathrm{MoS}_{2}$, while $J_{E C S A}$ increases faster in the case of $2 \mathrm{H} \mathrm{MoS}_{2}$. More importantly, the ECSA-normalized current density from $\mathrm{MoS}_{2}$ 
electrodes is almost one order of magnitude higher than that of the polycrystalline Pt electrode at $0.02 \mathrm{~mA} \mathrm{~cm}_{E C S A}^{-2}$ at the same a
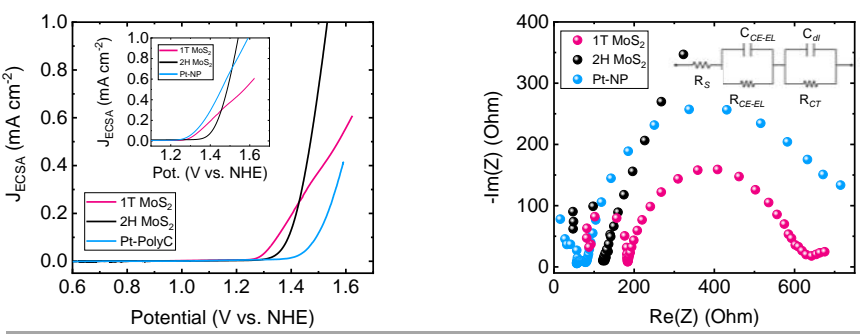

Figure 4. Comparison of the electrochemical responses from $\mathrm{MoS}_{2}$ nanosheets and platinum. (a) ECSA-normalized polarization curves for the DMS oxidation on $\mathrm{MoS}_{2}$ nanosheets and polycrystalline platinum (Pt-PolyC). Inset: Comparison between the $1 T^{\prime}$ and $2 \mathrm{H} \mathrm{MoS}$ nanosheets with supported Pt nanoparticles (Pt-NP). (b) Nyquist plots of the $1 T^{\prime}$ and $2 \mathrm{H} \mathrm{MoS}_{2}$ nanosheets as well as Pt nanoparticles measured at $1350 \mathrm{mV}$ vs. NHE.

applied potential (Figure 4a). $\mathrm{MoS}_{2}$ also compares favorably with Pt nanoparticles that exhibit a current density of $0.28 \mathrm{~mA}$ $c m_{E C S A}^{-2}$ for the reaction (Inset Figure 4a). We performed electrochemical impedance spectroscopy (EIS) on electrodes of the different phases of $\mathrm{MoS}_{2}$ as well as Pt nanoparticles (Figure 4b). The EIS measurements were performed at onset potentials of 1300 and $1350 \mathrm{mV}$ vs. NHE. The impedance responses consist of 2 semi-circles corresponding to the electrolyte response for the highest frequencies and the reaction responses for the lower frequencies. The high frequency responses in the form of quasi semi-circles are independent of the applied potential at the working electrode and are attributed to the responses from the counter electrode, substrate-catalyst interface and the electrolyte. ${ }^{37}$ The second semi-circle originates from the working electrode and originated from the charge transfer resistance $\left(R_{C T}\right)$ and the capacitances. The equivalent circuit used for fitting the EIS data is shown in inset of Figure $4 \mathrm{~b}$. The values of $R_{C T}$ decrease when the applied potential is increased, reflecting improved reaction kinetics (Figure S8a, b and Table S1). At $1300 \mathrm{mV}$ vs. NHE, the charge transfer resistance is estimated to be $\sim 370 \Omega$ for $1 T^{\prime} \mathrm{MoS}_{2}$, which is lower than the values extracted for $2 \mathrm{H} \mathrm{MoS}_{2}(1480 \Omega)$ and Pt nanoparticles ( $790 \Omega)$.

\section{Quantification of DMSO production and estimation of Faradic efficiency}

Next, we investigated the product of the DMS oxidation reaction by holding the potential of the electrode at $1500 \mathrm{mV}$ vs. NHE. The products of the reaction after 4,8 and 24 hours were analyzed using nuclear magnetic resonance (See "Detection of the products of the reaction via Nuclear magnetic resonance (NMR)" section in the Supporting Information). According to the literature, the oxidation of DMS can form several products including dimethyl sulfoxide, dimethyl sulfone and methyl sulfonates. ${ }^{38,39}$ In our NMR measurements, DMS and DMSO signatures are clearly identified at $2.08 \mathrm{ppm}$ and 2.5 ppm respectively (Figure $5 \mathbf{5}$ ). The NMR peaks from dimethyl sulfone and methyl sulfonates - expected at 2.91 and $\sim 3.1 \mathrm{ppm}$ - are not detected even after 24 hours of reaction (Figure S10). These results demonstrate that the electrochemical oxidation of DMS on the MoS 2 nanozymes is highly selective towards the formation of DMSO. We note that as the reaction proceeds, the DMSO signals increase continuously with time while the signatures for DMS decrease. Figure $\mathbf{5 b}$ shows the production of DMSO over 24 hours from $\mathrm{MoS}_{2}$ and platinum electrodes. The production of DMSO from the $1 \mathrm{~T}^{\prime}$ phase of $\mathrm{MoS}_{2}$ is largely maintained up to 24 hours with a total production of $250 \mathrm{~mol} \mathrm{~g}$ 1 , equivalent to a production rate of $680 \mathrm{~L} \mathrm{~h}^{-1}$ per gram of catalyst (Inset Figure $5 \mathrm{~b}$ ). This performance is clearly higher than that of $2 \mathrm{H} \mathrm{MoS} 2$ and Pt nanoparticles that exhibit a production of 30 and $71 \mathrm{~mol} \mathrm{~g}^{-1}$ after 24 hours equivalent to $\approx$ $89 \mathrm{~L} \mathrm{~h}^{-1} \mathrm{~g}^{-1}$ and $\approx 210 \mathrm{~L} \mathrm{~h}^{-1} \mathrm{~g}^{-1}$, respectively. More importantly our results also demonstrate that $1 T^{\prime} \mathrm{MoS}_{2}$ can sustain high production rate of DMSO with a quasi linear increase of the evolved DMSO, while the production rates from $\mathrm{Pt}$ and $2 \mathrm{H} \mathrm{MoS}_{2}$ tend to stabilize after 4 hours of reaction. This demonstrates that $1 \mathrm{~T}^{\prime} \mathrm{MoS}_{2}$ does not suffer from rapid deactivation of its catalytic properties compared to Pt or $2 \mathrm{H} \mathrm{MoS}_{2}$.

To obtain fundamental insight on the mechanisms of the reaction, we have estimated the quantity of electrons involved

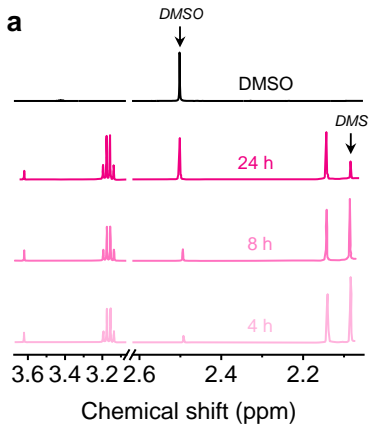

b

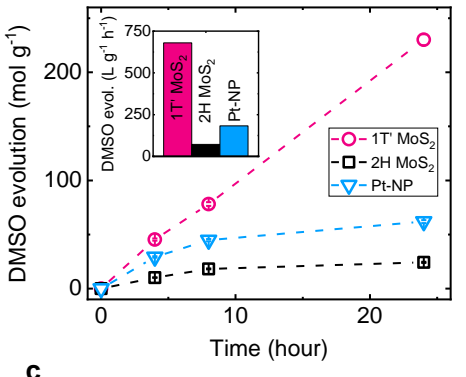

C

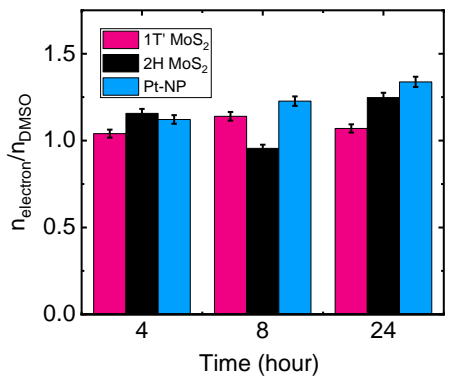

Figure 5. Production ratio of DMSO and determination of the reaction efficiency. (a) NMR spectra of the electrolyte during the reaction after 4,8 and 24 hours. The DMSO and DMS signatures are visible at $2.1 \mathrm{ppm}$ and $2.5 \mathrm{ppm}$ respectively. (b) DMSO production from the $1 T^{\prime}$ and $2 \mathrm{H} \mathrm{MoS}_{2}$ electrodes compared with supported Pt nanoparticles. Inset: Production rates of DMSO for the different electrodes averaged over 24 hours. (c) Ratio: $n_{e}^{-}: n_{D M S O}$ obtained from the NMR results and 
the electrochemical measurements for the 3 different electrodes after 4,8 and 24 hours.

per molecule of DMSO formed. We found that the ratio: $n_{e^{-}}$: $\mathrm{n}_{\mathrm{DMSO}}$ is the slowest for $1 \mathrm{~T}^{\prime} \mathrm{MoS}_{2}$ confirming the high efficiency of the $1 \mathrm{~T}^{\prime}$ phase. That is, after 24 hours, both $1 \mathrm{~T}^{\prime}$ and $2 \mathrm{H}$ phase demonstrate a lower $\mathrm{n}_{\mathrm{e}}{ }^{-}$: $\mathrm{n}_{\mathrm{DMSO}}$ ratio than $\mathrm{Pt}$ nanoparticles with 1.07 and 1.25 electrons per DMSO molecule, respectively, compared to 1.34 for Pt nanoparticles. Assuming one electron involved in the reaction, the Faradaic efficiency for the initial 4 hours of reaction is close to $96.2 \pm 2.1 \%$ for $1 \mathrm{~T}^{\prime}$ phase $\mathrm{MoS}_{2}$ and $86.4 \pm 1.8$ for the $2 \mathrm{H} \mathrm{MoS}$ compared to $89.1 \pm 1.9 \%$ for $\mathrm{Pt}$ nanoparticles (Figure 5c). After 24 hours, the Faradaic efficiency (FE) is largely maintained for $1 T^{\prime} \mathrm{MoS}_{2}$ (97\%) and $2 \mathrm{H} \mathrm{MoS}_{2}(92$ \%) - in contrast to $\mathrm{Pt}$ nanoparticle catalysts where the $\mathrm{FE}$ retention drops to $83.8 \%$. To confirm the stability of the $\mathrm{MoS}_{2}$ nanosheets, the electrodes were characterized using Raman and XPS spectroscopy (Figure S11) ${ }^{40,41}$. The signatures from the $1 T^{\prime}$ phase are clearly visible from the $E_{1 g}, J_{1}$ and $J_{3}$ peaks as well as from the deconvolution of the Mo3d and S2p XPS spectra. Importantly virtually no signals from the oxidized Mo and $\mathrm{S}$ are detected in XPS, while the $1 T^{\prime}$ phase is estimated to $\sim 50 \%$ after the reaction, suggesting minimal relaxation of the metallic structure.

\section{Estimation of intrinsic activity of $1 \mathrm{~T}^{\prime}$ and $2 \mathrm{H} \mathrm{MoS}_{2}$ nanozymes towards the oxidation of DMS}

To benchmark activity of $1 \mathrm{~T}^{\prime}$ and $2 \mathrm{H}$ phases of $\mathrm{MoS}_{2}$ with that of platinum towards oxidation of DMS, we normalized the activity per mass of catalysts loaded on the electrode. Figure 6 a shows the FE-normalized mass activity of $\mathrm{MoS}_{2}$ compared to $\mathrm{Pt}$ nanoparticles. At $1400 \mathrm{mV}$ vs. NHE, the activity of $1 \mathrm{~T}^{\prime} \mathrm{MoS}_{2}$ nanosheets reaches $240 \mathrm{~A} \mathrm{~g}^{-1}$ - compared to $16.4 \mathrm{~A} \mathrm{~g}^{-1}$ for $2 \mathrm{H}$

a

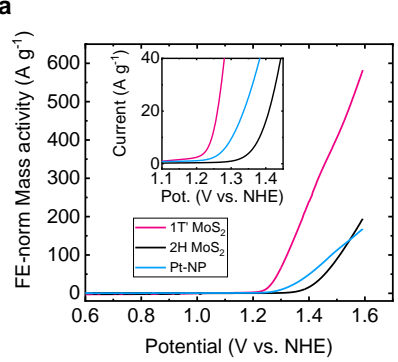

c

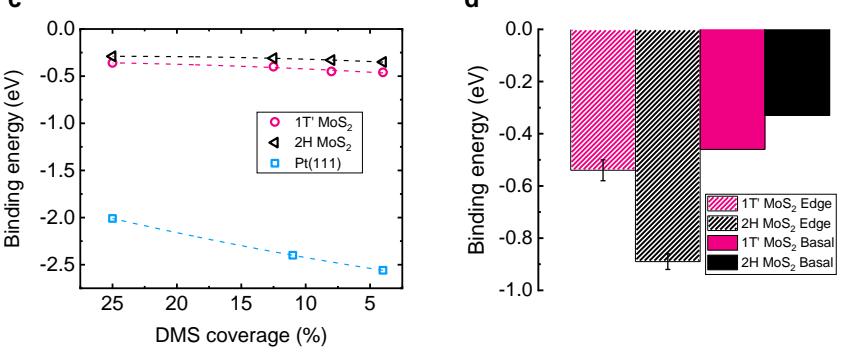

Figure 6. Estimation of the electrocatalytic performance of $\mathrm{MOS}_{2}$ nanosheets towards DMSO oxidation. (a) Evolution of the mass activity (in $\mathrm{A}^{-1}$ ) with the increase of the applied potential for $1 T^{\prime}$ and $2 \mathrm{H} \mathrm{MoS}_{2}$ electrodes compared to $20 \%$ $P t / C$. The mass activity is normalized by the faradaic efficiency. (b) Evolution of the Turnover frequency (TOF) with the onsetpotential for $1 \mathrm{~T}^{\prime}$ and $2 \mathrm{H} \mathrm{MoS} \mathrm{S}_{2}$ nanosheets and compared to polycrystalline Pt. (c) Binding energy of DMS on the surface of $\mathrm{MoS}_{2}$ and Pt (111) as a function of the coverage fraction. (d) Comparison between the binding energy of DMS at the Mo $100 \%$ S-edges and the basal planes of the $1 T^{\prime}$ and $2 \mathrm{H}$ phases of $\mathrm{MOS}_{2}$.

$\mathrm{MoS}_{2}$ nanosheets. The DMS oxidation performance of the $1 \mathrm{~T}^{\prime}$ polymorph is 5-fold larger than for Pt nanoparticles supported on carbon. The required potential to generate a current of $50 \mathrm{~A}$ $\mathrm{g}^{-1}$ (equivalent to $132 \mathrm{~L} \mathrm{~g}^{-1} \mathrm{~h}^{-1}$ ) is $1280 \mathrm{mV}$ vs. $\mathrm{NHE}-120 \mathrm{mV}$ lower than in the case of Pt nanoparticles. To further evaluate the intrinsic electrocatalytic properties of the $\mathrm{MoS}_{2}$ nanosheets, we estimated the turnover frequency (TOF) based on ECSA measurements and assuming active site density of $1.5 \times 10^{15}$ $\mathrm{cm}^{-2}$ for a flat $\mathrm{MoS}_{2}$ surface. We note that for the calculations of the TOF, we assume that the whole surface of the $\mathrm{MoS}_{2}$ nanosheets is active. The TOF values from the $1 \mathrm{~T}^{\prime}$ and $2 \mathrm{H}$ phases were compared to those from the platinum nanoparticles (Figure 6b). The density of active sites for platinum was estimated from UPD measurements and assuming a site density of $1.1 \times 10^{15} \mathrm{~cm}^{-2} .42$ The higher intrinsic activity from the $\mathrm{MoS}_{2}$ nanosheets compared to $\mathrm{Pt}$ is clearly visible from the TOF values. When compared to platinum nanoparticles, $\mathrm{MoS}_{2}$ nanosheets demonstrated excellent performance with notably lower onset potential and similar TOF values at lower applied potentials. A TOF of $0.1 \mathrm{~s}^{-1}$ is achieved for an applied potential of $1258 \mathrm{mV}$ vs. NHE in the case of $1 \mathrm{~T}^{\prime} \mathrm{MoS}_{2}$. This potential is 73 $\mathrm{mV}$ and $180 \mathrm{mV}$ smaller than the potential for achieving similar activity from $2 \mathrm{H} \mathrm{MoS} 2$ and $\mathrm{Pt}$ nanoparticles, respectively.

To gain further understanding on the origin of the high activity of $\mathrm{MoS}_{2}$ towards DMS oxidation, we computed the binding energy ( $E_{b}$ in $e V$ ) of DMS adsorption for different levels of DMS coverage on $\mathrm{MoS}_{2}$ and $\mathrm{Pt}(111)$ surfaces using density functional theory (See "Calculation of the binding energy $\left(E_{b}\right)$ of DMS" section in Supporting Information and Figure S12). Figure 6c shows the binding energy of DMS on $\mathrm{MoS}_{2}$ and Pt surfaces as a function of the coverage fraction. In the dilute limit, we found that approximate potentials of 0.46 and $2.56 \mathrm{eV}$ are required to desorb DMS from $\mathrm{MoS}_{2}$ and $\mathrm{Pt}$, respectively. Charge density differences and Bader charge analysis show that DMS is charge donor in both cases and that the charge transfer (from DMS to the surface) is of $+0.18 \mathrm{e}$ and $+0.38 \mathrm{e}$ when DMS is adsorbed on $\mathrm{MoS}_{2}$ and $\mathrm{Pt}$, respectively (Figure S13). Moreover, the shortest distance between an atom of the DMS and an atom of the catalyst is $3.0 \AA$ and $2.3 \AA$ for $\mathrm{MoS}_{2}$ and $\mathrm{Pt}$, respectively. These key figures demonstrate the strong interaction between DMS and Pt by contrast to mild physisorption of DMS on $\mathrm{MoS}_{2}$. Interestingly, we found the bonding energy to be -0.55 and $0.90 \mathrm{eV}$ at the edges of the $1 \mathrm{~T}^{\prime}$ and $2 \mathrm{H}$ phases respectively (Figure 6d and Table S2). Our DFT calculations suggest that DMS preferentially physisorbs at both the edges and the surface sites of the $1 \mathrm{~T}^{\prime} \mathrm{MoS}_{2}$ nanosheets, leading to more efficient oxidation to DMSO. We anticipate that the strong DMS adsorption in the case of the $2 \mathrm{H}$ phase lowers the performance and stability of the $2 \mathrm{H}$ phase. Our DFT calculations combined with the examination of the structure of the DMSOR demonstrate that the superior activity from the $\mathrm{MoS}_{2}$ nanosheets originates from its biomimetic structure and composition. Overall, our results reveal the superior activity of the $1 \mathrm{~T}^{\prime}$ phase toward the oxidation of organic sulfide. The activation of the $\mathrm{MoS}_{2}$ nanosheets originates from the lower binding energy of the 
DMS compared to that of platinum. The $1 T^{\prime}$ phase of $\mathrm{MoS}_{2}$ is expected to be active from both the edges and the basal planes while the active sites from the $2 \mathrm{H}$ phase are localized only on the basal planes due to the larger binding energy on the $2 \mathrm{H}$ edge sites. Further calculations of the free energy of the overall DMS oxidation reaction that would provide additional information on the reaction pathways are currently ongoing in our group, but are beyond the scope of this study. Besides the thermodynamics of the reaction, the metallic nature of the $1 \mathrm{~T}^{\prime}$ phase of $\mathrm{MoS}_{2}$ is expected to further improve the kinetics of the reaction and is responsible for the reduction of the overpotentials. Similar behavior has previously been reported in the case of the hydrogen evolution reaction on the $1 \mathrm{~T}^{\prime}$ phase of group-6 TMDs. ${ }^{35}$ This is supported by the reduced Tafel slope of $75 \mathrm{mV} \mathrm{dec}^{-1}$ and the low charge transfer resistance $R_{\text {Cт }}$ of 370 $\Omega$.

\section{Role of defects and crystallinity of $\mathrm{MoS}_{2}$ on the DMS oxidation}

Defect engineering of $\mathrm{MoS}_{2}$ has been explored to trigger the electrocatalytic reactions such as the hydrogen evolution reaction or the splitting water. ${ }^{43,44}$ To further understand the origin of the $\mathrm{MoS}_{2}$ activity toward the oxidation of DMS, we prepared electrodes with increasing density of defects. The defect concentration was tuned by thermally annealing $\mathrm{MoS}_{2}$ under hydrogen atmosphere at increasing temperatures in order to create point-defect vacancies $\left(2 \mathrm{H} \mathrm{MoS}_{2}-\mathrm{Vs}\right)$ and sulfur stripping defects $\left(2 \mathrm{H} \mathrm{MoS}_{2}\right.$-Ss) corresponding to low and high defect densities respectively. ${ }^{45}$ We also prepared amorphous $\mathrm{MoS}_{2}\left(\mathrm{~A}-\mathrm{MoS}_{\mathrm{x}}\right)$ nanosheets grown under hydrothermal conditions in DMF (See details in Supporting Information). The structure and the composition of the $\mathrm{MoS}_{2}$ electrodes were analyzed by using Raman and XPS spectroscopy (Figure S16). The sulfur-to-molybdenum ratios were estimated to 2.24, 1.79 and 0.63 for $\mathrm{A}-\mathrm{MoS}_{\mathrm{x}}, 2 \mathrm{H} \mathrm{MoS}-\mathrm{V}_{\mathrm{S}}$ and $2 \mathrm{H} \mathrm{MoS}_{2}-\mathrm{S}_{\mathrm{s}}$ respectively. Figure $7 \mathbf{a}, \mathbf{b}$ shows the DMS oxidation performance of the different electrodes compared with the $1 \mathrm{~T}^{\prime}$ and $2 \mathrm{H}$ phases of $\mathrm{MoS}_{2}$. We also estimated the electrochemically active surface area (ECSA) by measuring the electrochemical double layer capacitance. The corresponding ECSA-normalized polarization curves are presented in Figure 7c. The geometrical current density is found to be the highest for A-MoS 2 along with $1 \mathrm{~T}^{\prime}$ $\mathrm{MoS}_{2}$, while the activity of the $2 \mathrm{H}$ phase increases with the concentration of sulfur vacancies. Conversely, JECSA sharply decreases for $A-M_{0} S_{x}$ suggesting that the large geometrical current density is ascribed to the more porous or rough nature of the materials. Our results also demonstrate that the performance from defective $2 \mathrm{H} \mathrm{MoS}_{2}$ is comparable to that of its pristine counterpart. We further calculated the binding energy of DMS on the basal plane of $1 \mathrm{~T}^{\prime}$ and $2 \mathrm{H} \mathrm{MoS}_{2}$ with single sulfur vacancies (Figure S13, Table S2) and found that, for the same phase, the thermodynamics of the adsorption of DMS is virtually identical on defect-free $\mathrm{MoS}_{2}$ and $\mathrm{MoS}_{2}-\mathrm{V}_{\mathrm{S}}$ basal planes.

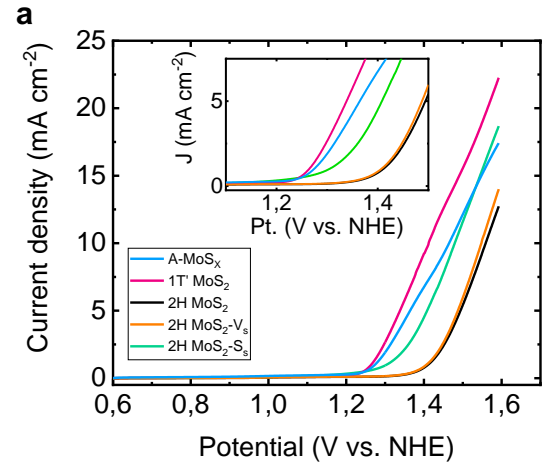

b

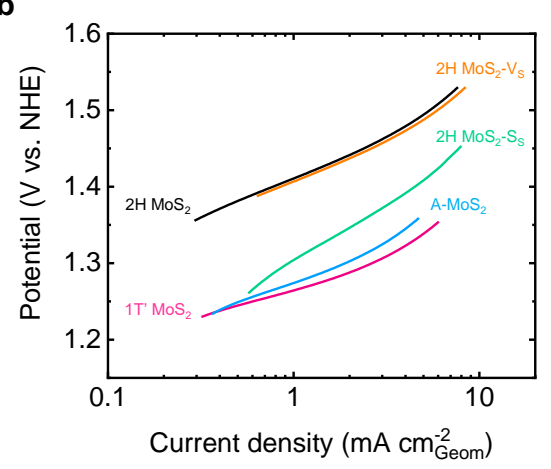

C

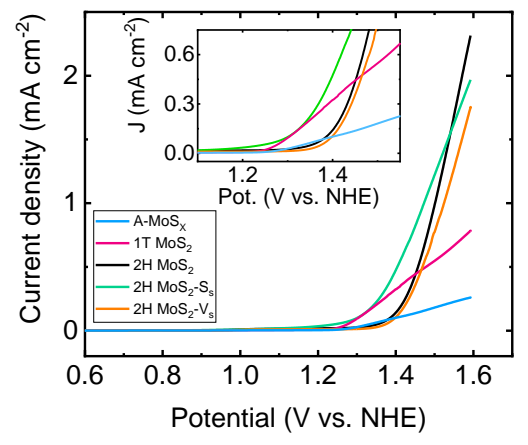

Figure 7. Electrocatalytic behavior of amorphous $M_{0} S_{x}$ and defective MoS electrodes towards the oxidation of DMS. $(a, b)$ Polarization curves (a) and corresponding Tafel plots (b) of the different molybdenum sulfide electrodes. Scan rate: $20 \mathrm{mV} \mathrm{s}^{-1}$. (c) ECSA-normalized (J $J_{\text {ECSA }}$ ) polarization curves of amorphous MoS and defective $\mathrm{MOS}_{2}$ electrodes toward the oxidation of DMS compared to the pristine $1 T^{\prime}$ and $2 \mathrm{H}$ phases of $\mathrm{MoS}_{2}$. Inset: Magnification of the low potential region.

\section{DMS oxidation mechanism from $1 T^{\prime}$ MoS $_{2}$ nanosheets}

Previous reports on the oxidation of DMS have suggested that the reaction proceeds through a one (DMSO) or two electrons (DMSO${ }_{2}$ ) processes. ${ }^{38,46,47}$ Instead, our results demonstrate that the DMS is selectively electrochemically oxidized into DMSO via a one-electron reaction on both $\mathrm{Pt}$ and $\mathrm{MoS}_{2}$. We anticipate that the oxidation of DMS involves the formation of a radical cation after the electron withdrawal from DMS: $\left(\mathrm{CH}_{3}\right)_{2} \mathrm{~S} \rightarrow$ $\left(\mathrm{CH}_{3}\right)_{2} \mathrm{~S}^{++}+e^{-}$as proposed by Elinson and Simonet. ${ }^{23}$ The first hint of the presence of radicals during the DMS oxidation is brought by the appearance of light-yellow color in the electrolyte in absence of oxygen during the reaction (Figure S17). To further point out the role of radicals during the 
reaction, we performed electron paramagnetic resonance (EPR) to detect the formation of sulfur radicals as reaction intermediates. The electro-oxidation of DMS was performed in the presence and in the absence of a radical trapping agent: 5,5Dimethyl-1-pyrroline N-oxide, DMPO (See "EPR measurements" section in the Supporting Information). First, we performed the reaction in the absence of DMS and no free radical signals were detected. In the presence of DMS, clear EPR signatures are identified centered around $3510 \mathrm{G}$ and the intensity of the signals increases with time (Figure 8a). The EPR measurements after $30 \mathrm{~min}$ revealed a six-line spectrum different from the spectrum of oxidized radical trapping agent ruling out any oxidation of DMPO. ${ }^{48}$ The spectrum corresponds to hyperfine coupling constants of $\mathrm{a}_{\mathrm{N}}=15.4 \mathrm{G}, \mathrm{a}_{\mathrm{H}}=21 \mathrm{G}(\mathrm{g}=2.0621)$ - close

a
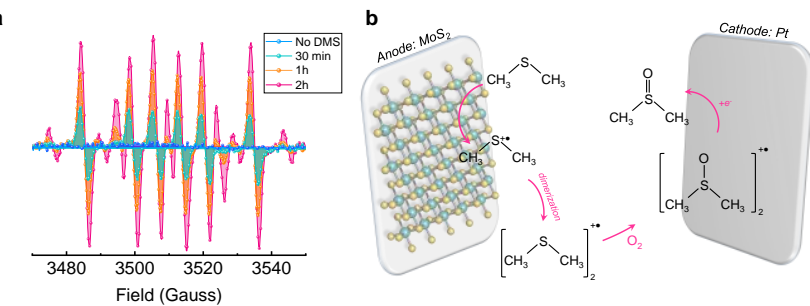

c
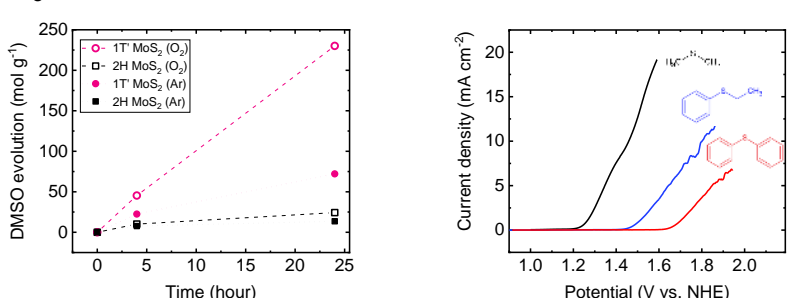

Potential (V vs. NHE)

Figure 8. Mechanism of the electrochemical oxidation of DMS. (a) EPR spectra from the electrolyte in presence of radical trapping agent (DMPO) with absence of DMS (black, blank experiment) and after $30 \mathrm{~min}$ (orange), $1 \mathrm{~h}$ (blue) with presence of DMS. (b) Proposed mechanism for the reaction of DMS oxidation in $\mathrm{O}_{2}$ saturated solution. (c) Comparison of the DMSO production from $1 T^{\prime}$ and $2 \mathrm{H} \mathrm{MoS}_{2}$ in presence or absence of $\mathrm{O}_{2}$ dissolved in the electrolyte.

to the constants obtained when trapping $\mathrm{CH}_{3} \mathrm{~S}^{--}$using DMPO: $\mathrm{a}_{\mathrm{N}}=15.03 \mathrm{G}$ and $\mathrm{a}_{\mathrm{H}}=18 \mathrm{G} .{ }^{49}$ Our EPR investigation thus points out that the oxidation of DMS involves the formation of an alkyl sulfur radical that further reacts with DMPO. The additional signals detected after $1 \mathrm{~h}$ are attributed to the reduction of the DMPO $\left(a_{N}=14.5 \mathrm{G}, a_{H}=19.5 \mathrm{G}\right.$, see details of the reaction mechanism in the Supporting Information). ${ }^{50}$ The sulfur radical cations formed on $\mathrm{MoS}_{2}$ nanosheets thus act as a "cation pool" in the formation of DMSO as observed for other anodic oxidation reactions in electrosynthesis. ${ }^{51,52} \mathrm{We}$ propose that the sulfur radicals then reacts with the radical superoxide $\mathrm{O}_{2}^{-}$. formed at the cathode as previously proposed for the oxidation of thioethers (Figure $\mathbf{8 b}$ and Figure S19). ${ }^{53}$ To further confirm the role of oxygen in the reaction, we performed the same reaction under a flow of argon instead of oxygen. After 24 hours, the production rate of DMSO from the $1 \mathrm{~T}^{\prime}$ and $2 \mathrm{H} \mathrm{MoS}_{2}$ nanozymes decreases from 680 and $71 \mathrm{~L} \mathrm{~h}^{-1} \mathrm{~g}^{-1}$ to only 213 and $38 \mathrm{~L} \mathrm{~h}^{-1} \mathrm{~g}^{-1}$ for the $1 \mathrm{~T}^{\prime}$ and $2 \mathrm{H} \mathrm{MoS}_{2}$ respectively (Figure $8 \mathrm{c}$ ). We also found that the Faradic efficiency decreased at higher applied potential (i.e. at higher current density) due to the

limited concentration of $\mathrm{O}_{2}$ dissolved in the electrolyte. To generalize our approach, $\mathrm{MoS}_{2}$ nanosheets have been tested toward the oxidation of different alkyl and aryl sulfide molecules. Our investigations show that ethyl phenyl sulfide and diphenyl sulfide can be oxidized using $1 \mathrm{~T}^{\prime} \mathrm{MoS}_{2} 2 \mathrm{D}$ nanozymes and the onsetpotential associated with the reaction is found to increase when replacing alkyl chains for aryl groups revealing differences in term of reactivity (Figure $\mathbf{8 d}$ ).

\section{Conclusions}

Our findings reveal the potential of phase engineered TMDs as biomimetic electrocatalysts for the electrochemical oxidation of sulfide-based molecules. We identified the exfoliated $\mathrm{MoS}_{2}$ nanosheets as efficient two-dimensional electrocatalysts for the oxidation of alkyl and aryl sulfides. Remarkably, the metallic $1 T^{\prime}$ phase of $\mathrm{MoS}_{2}$ exhibits superior activity towards the production of DMSO from DMS compared to noble metals such as platinum nanoparticles and dirhodium complexes with reduced onset potential and lower Tafel slope. By carefully estimating the density of active sites, we determined that the TOF reaches $0.87 \mathrm{~s}^{-1}$ at a potential of $1400 \mathrm{mV}$ vs. NHE, 30-fold larger than for polycrystalline platinum $\left(\approx 0.03 \mathrm{~s}^{-1}\right)$. The reaction proceeds via one-electron transfer and the Faradaic efficiency is found to be stable for at least 24 hours. We believe that our results open avenues for novel applications of TMDs towards electrochemical-assisted synthesis of organic molecules.

\section{Conflicts of interest}

There are no conflicts to declare.

\section{Acknowledgements}

D.V., L.M. and K.Q. acknowledge funding from the European Research Council (ERC) under the European Union's Horizon 2020 research and innovation programme (grant agreement No 804320). D.V. and Z.Q. acknowledge financial support from the US Army RDECom Grant NW911NF-17-2-0033. H.E.M. acknowledges the French embassy in Egypt. N.O. thanks The Hong Kong Polytechnic University and the department of Applied Physics for the computational resources. L.L. acknowledges National Natural Science Foundation of China (21978111) and Zhejiang Provincial Natural Science Foundation of China (LY19B030005).

\section{Notes and references}

1 P. K. Robinson, Essays Biochem, 2015, 59, 1-41.

2 J. R. Knowles, Nature, 1991, 350, 121-124.

3 S. J. Benkovic and S. Hammes-Schiffer, Science, 2003, 301, 1196-1202.

4 J. M. Le and K. L. Bren, ACS Energy Lett., 2019, 4, 2168-2180. 
5 B. Hinnemann, P. G. Moses, J. Bonde, K. P. Jørgensen, J. H. Nielsen, S. Horch, I. Chorkendorff and J. K. Nørskov, J. Am. Chem. Soc., 2005, 127, 5308-5309.

6 H. Wei and E. Wang, Chem. Soc. Rev., 2013, 42, 6060-6093.

7 J. Wu, X. Wang, Q. Wang, Z. Lou, S. Li, Y. Zhu, L. Qin and H. Wei, Chem. Soc. Rev., 2019, 48, 10041076.

8 L. Gao, J. Zhuang, L. Nie, J. Zhang, Y. Zhang, N. Gu, T. Wang, J. Feng, D. Yang, S. Perrett and X. Yan, Nature Nanotech, 2007, 2, 577-583.

9 F. Natalio, R. André, A. F. Hartog, B. Stoll, K. P. Jochum, R. Wever and W. Tremel, Nature Nanotech, 2012, 7, 530-535.

10 Q. Wang, X. Zhang, L. Huang, Z. Zhang and S. Dong, Angew. Chem. Int. Ed. Engl., 2017, 56, 16082-16085.

11 Y. Liu, D. L. Purich, C. Wu, Y. Wu, T. Chen, C. Cui, L. Zhang, S. Cansiz, W. Hou, Y. Wang, S. Yang and W. Tan, J. Am. Chem. Soc., 2015, 137, 1495214958.

12 Y. Lin, J. Ren and X. Qu, Acc. Chem. Res., 2014, 47, 1097-1105.

13 S. Ghosh, P. Roy, N. Karmodak, E. D. Jemmis and G. Mugesh, Angew. Chem. Int. Ed. Engl., 2018, 57, 4510-4515.

14 D. Voiry, H. S. Shin, K. P. Loh and M. Chhowalla, Nature Reviews Chemistry, 2018, 2, 0105.

15 D. Voiry, J. Yang and M. Chhowalla, Advanced Materials, 2016, 28, 6197-6206.

16 X. J. Chua, J. Luxa, A. Y. S. Eng, S. M. Tan, Z. Sofer and M. Pumera, ACS Catal., 2016, 6, 57245734.

17 B. Mohanty, M. Ghorbani-Asl, S. Kretschmer, A. Ghosh, P. Guha, S. K. Panda, B. Jena, A. V.

Krasheninnikov and B. K. Jena, ACS Catal., 2018, 8, 1683-1689.

18 T. J. Johnson and D. D. Hedge, Am J Health Syst Pharm, 2002, 59, 1333-1339.

19 K. P. Garnock-Jones, S. Dhillon and L. J. Scott, CNS Drugs, 2009, 23, 793-803.

20 E. G. Mata, Phosphorus, Sulfur, and Silicon and the Related Elements, 1996, 117, 231-286.

21 C. O. Kinen, L. I. Rossi and R. H. de Rossi, J. Org. Chem., 2009, 74, 7132-7139.

22 P. T. Cottrell and C. K. Mann, J. Electrochem. Soc., 1969, 116, 1499-1503.

23 M. N. Elinson and J. Simonet, Journal of Electroanalytical Chemistry, 1992, 336, 363-367.

24 J. V. Lauritsen, M. V. Bollinger, E. Lægsgaard, K. W. Jacobsen, J. K. Nørskov, B. S. Clausen, H. Topsøe and F. Besenbacher, Journal of Catalysis, 2004, 221, 510-522.
25 X. Chen, C. McGlynn and A. R. McDonald, Chem. Mater., 2018, 30, 6978-6982.

26Z. Chen, C. Liu, J. Liu, J. Li, S. Xi, X. Chi, H. Xu, I.H. Park, X. Peng, X. Li, W. Yu, X. Liu, L. Zhong, K. Leng, W. Huang, M. J. Koh and K. P. Loh, Advanced Materials, 2020, 32, 1906437.

27 P. M. Wood, FEBS Letters, 1981, 124, 11-14.

28 P. Joensen, R. F. Frindt and S. R. Morrison, Materials Research Bulletin, 1986, 21, 457-461.

29 G. Eda, H. Yamaguchi, D. Voiry, T. Fujita, M. Chen and M. Chhowalla, Nano Lett., 2011, 11, 5111-5116.

30 G. Eda, T. Fujita, H. Yamaguchi, D. Voiry, M. Chen and M. Chhowalla, ACS Nano, 2012, 6, 7311-7317.

31 D. Voiry, M. Salehi, R. Silva, T. Fujita, M. Chen, T. Asefa, V. B. Shenoy, G. Eda and M. Chhowalla, Nano Lett., 2013, 13, 6222-6227.

32 J. Pu, Y. Yomogida, K.-K. Liu, L.-J. Li, Y. Iwasa and T. Takenobu, Nano Lett., 2012, 12, 4013-4017.

33 M. Acerce, D. Voiry and M. Chhowalla, Nature Nanotechnology, 2015, 10, 313-318.

34 L. Zhao, H. Zhang and Y. Wang, J. Org. Chem., 2016, 81, 129-136.

35 D. Voiry, R. Fullon, J. Yang, C. de C. C. e Silva, R. Kappera, I. Bozkurt, D. Kaplan, M. J. Lagos, P. E. Batson, G. Gupta, A. D. Mohite, L. Dong, D. Er, V. B. Shenoy, T. Asefa and M. Chhowalla, Nat Mater, 2016, 15, 1003-1009.

36 C. L. Green and A. Kucernak, J. Phys. Chem. B, 2002, 106, 1036-1047.

37 L. Tao, Z. Huo, Y. Ding, Y. Li, S. Dai, L. Wang, J. Zhu, X. Pan, B. Zhang, J. Yao, M. K. Nazeeruddin and M. Grätzel, J. Mater. Chem. A, 2015, 3, 2344 2352.

38 P. T. Cottrell and C. K. Mann, J. Electrochem. Soc., 1969, 116, 1499-1503.

39 I. Barnes, J. Hjorth and N. Mihalopoulos, Chem. Rev., 2006, 106, 940-975.

40 G. Liu, Z. Li, J. Shi, K. Sun, Y. Ji, Z. Wang, Y. Qiu, Y. Liu, Z. Wang and P. Hu, Applied Catalysis B: Environmental, 2020, 260, 118134.

41 Y. Zhang, H. Sun, Y. Qiu, X. Ji, T. Ma, F. Gao, Z. Ma, B. Zhang and P. Hu, Carbon, 2019, 144, 370381.

42 T. F. Jaramillo, K. P. Jørgensen, J. Bonde, J. H. Nielsen, S. Horch and I. Chorkendorff, Science, 2007, 317, 100-102.

43 J. Xie, H. Zhang, S. Li, R. Wang, X. Sun, M. Zhou, J. Zhou, X. W. (David) Lou and Y. Xie, Advanced Materials, 2013, 25, 5807-5813.

44 H. Li, C. Tsai, A. L. Koh, L. Cai, A. W. Contryman, A. H. Fragapane, J. Zhao, H. S. Han, H. C. Manoharan, F. Abild-Pedersen, J. K. Nørskov and X. Zheng, Nat Mater, 2016, 15, 48-53. 
45 L. Li, Z. Qin, L. Ries, S. Hong, T. Michel, J. Yang, C. Salameh, M. Bechelany, P. Miele, D. Kaplan, M. Chhowalla and D. Voiry, ACS Nano, 2019, 13, 6824-6834.

46 M. N. Elinson and J. Simonet, Journal of Electroanalytical Chemistry, 1992, 336, 363-367.

47 M. D. Ryan, J. Yau and M. Hack, J. Electrochem. Soc., 1997, 144, 1952-1957.

48 J. Marcon, G. Mortha, N. Marlin, F. Molton, C.

Duboc and A. Burnet, Holzforschung, 2017, 71, 599610.

49 M. J. Davies, L. G. Forni and S. L. Shuter, ChemicoBiological Interactions, 1987, 61, 177-188.

50 Y. Gorbanev, R. Soriano, D. O'Connell and V. Chechik, $J$ Vis Exp, , DOI:10.3791/54765.

51 J. Yoshida, A. Shimizu and R. Hayashi, Chem. Rev., 2018, 118, 4702-4730.

52 R. Hayashi, A. Shimizu and J. Yoshida, J. Am. Chem. Soc., 2016, 138, 8400-8403.

53 C. Ye, Y. Zhang, A. Ding, Y. Hu and H. Guo, Scientific Reports, 2018, 8, 1-6. 